\title{
Study on collaborative management of sustainable supply chain in water diversion Project
}

\section{Yali LU ( $\nabla$ luyali676@163.com )}

School of management and economics,North China University of Water Resources and Electric Power, Zhengzhou City, Henan Province

\section{Meng LIU}

College of Information Engineering, Zhengzhou University of Industrial Technology

\section{Wenwen Ding}

School of Management and Economics, North China University of Water Resources and Electric Power, Zhengzhou City, Henan Province

\section{Research Article}

Keywords: Water Diversion Project, Sustainable Supply Chain,collaboration

Posted Date: February 16th, 2022

DOI: https://doi.org/10.21203/rs.3.rs-1342303/v1

License: (1) (1) This work is licensed under a Creative Commons Attribution 4.0 International License. Read Full License 


\title{
Study on collaborative management of sustainable supply chain in water
}

diversion Project

\author{
Meng Liu ${ }^{1}$, Wenwen Ding ${ }^{2}$, Yali Lu ${ }^{2 *}$ \\ 1. College of Information Engineering, Zhengzhou University of Industrial
} Technology, 450064;

2. School of Management and Economics,North China University of Water Resources and Electric Power, Zhengzhou City, Henan Province, 450046;

* Corresponding author. E-mail address: luyali676@163.com (Ya li LU).

Abstract Measuring the collaboration of the sustainable supply chain of water diversion projects is of great significance to the sustainable operation of the project. To achieve this, it needs to develop a scientific and reasonable collaboration measurement method. Given the economic, environmental and social benefits of water diversion projects, and based on the theory of the sustainable supply chain, an order parameter index system for collaboration measurement was constructed in this study. The Dematel and Analytic Network Process methods were used to compute the local and global weights of each order parameter. A total of 161 valid questionnaires were collected from residents along the South-to-North Water Diversion Project Middle Route to measure its collaboration from 2018 to 2020 . Results showed that the orderliness of the four flow subsystems of the South-to-North Water Diversion Project Middle Route increases year by year. Compared with the other three flow subsystems, the knowledge flow subsystem has a lower level of orderliness, suggesting that communication and 
exchanges among people along the route should be strengthened. Such findings can help identify the weak link in the collaborative management of the sustainable supply chain of water diversion projects and improve it in a targeted manner.

Keywords Water Diversion Project; Sustainable Supply Chain;collaboration

\section{Introduction}

Water diversion is one of the most effective ways to address the uneven distribution of water resources( $\mathrm{Gu}$ et al.,2017), but the problems brought about by it are becoming increasingly prominent, because such large-scale projects usually span regions and basins, involve a large number of stakeholders and many aspects of the society, and belong to quasi-public goods. Through field visits and research, the main problems faced by the current operation and management of water diversion projects are summarized as follows: (1) sensitive social issues arising from large-scale land acquisition and resident relocation for water diversion projects; (2) the adverse impact of water diversion on water sources and ecological environments4 along the route; and (3) the difficulty in coordination among multi-agent management departments and stakeholders. In response to these problems, Wang's team (Wang et al., 2004) first proposed applying the idea of supply chain management in the research of water diversion projects and achieved fruitful results. Based on their findings, many scholars have made further research, but most of them used the game theory and method to explore the coordination of interests among multiple agents involved in the supply chain of water diversion projects (Chen Z. S., 2012, 2013). Previous studies have shifted their focus to the economic, environmental and social benefits of the project 
from the main part of the project and economic benefits of stakeholders. This coincides with the original purpose of water diversion projects. The application of the sustainable supply chain theory and idea to water diversion projects has gradually become a new research hotspot. Li's team verified the feasibility of this proposal and constructed a structural model of the sustainable supply chain of water diversion projects ( $\mathrm{Li}$ et al., 2021). In addition, his team also employed collaborative management related theories, providing a new research perspective for the operation and management of similar large-scale projects.

The idea of collaborative management was first introduced into the field of business management in 1965 by the American strategic manager H. Igor Ansoff. Later, Haken elaborated the system theory in detail and called the synergetics a science of collaborative work. He believed that collaborative management is applicable to a wide range of fields from the natural sciences to the social sciences (Andrew et al., 2003; Kallis et al.,2009; Pirsoul et al.,2019). Although scholars remain divided over the connotation of collaborative management, but most of them have defined the collaborative management from the micro level to the macro level (German et al.,1984). The theory of collaborative management focuses on a complex system composed of a large number of subsystems with different properties(Berkes, 2017). The cooperation between subsystems may cause the self-organization of the whole system and lead to its evolution from a disorderly state to an orderly structure (Haken H., 2004). The collaborative management of the sustainable supply chain of water diversion projects is essentially a multi-agent cooperative process and a complex system containing a 
large number of subsystems (Li et al., 2021). The overall collaboration of a complex system and the mutual collaboration of its subsystems can be used to characterize the degree of harmony between subjects that are 'working together', providing an important reference for assessing the sustainable supply chain collaboration (SSCC) of water diversion projects.

The measurement of collaboration between subsystems involves a variety of industries, fields, and disciplines (Wang et al., 2020). For example, Lu constructed a composite system collaboration model to measure the orderliness and collaboration of each subsystem in the Beijing-Tianjin-Hebei region during 2008-2013 (Lu et al., 2015). Li constructed a composite system collaboration model combining technical innovation and ecological environment from the perspective of environmental protection, and studied three major city clusters in China using panel data (Li et al., 2016). Ma measured the orderliness and collaboration of economy in the Beijing-Tianjin-Hebei region by constructing a composite system collaboration model (Ma, 2019). Based on the composite system collaboration model, Gao used the science and technology statistics of Heilongjiang and Jilin to measure the current situation and future trends of the science and technology resource coordination in the Harbin-Changchun City Cluster, and designed an ecological governance mechanism for science and technology resources in the region (Gao et al., 2020). $\mathrm{Xu}$ and $\mathrm{Li}$ also established a composite system collaboration model based on order parameters (Li et al., 2021; Xu et al., 2013). In these studies, the collaboration among subsystems is mainly evaluated through the chain data envelopment analysis model (Xu et al., 2013), grey clustering method (Wang 
et al., 2020) and composite system collaboration model based on order parameters. The method of portraying the dynamic evolution process and laws of subsystems based on order parameters has been widely recognized by the academic community, and it also provides an important reference and basis for the research in this paper.

In summary, due to the public welfare nature of the water diversion project and the diversity of the management bodies along the route, the increase in the economic, environmental and social benefits of the project requires the collaboration of the management departments along the route. A scientific and reasonable evaluation of the collaboration along the route provides an important reference for enhancing the comprehensive benefits and management enthusiasm. Researchers have used different theories and models to conduct in-depth research on collaborative management at the government level, and some have constructed many methods and models to measure the degree of collaboration, providing an important reference for measuring the SSCC of water diversion projects. However, the authors believed that there is still room for improvement in previous studies. As for the management bodies of water diversion projects, scholars have mostly focused on the government level, and there are few studies that include enterprises and the public as management bodies. As for the application of the collaboration evaluation method, most scholars considered that the indicators were independent of each other, so they ignored the correlation between indicators when establishing the indicator system. Based on this, a sustainable supply chain model of water diversion projects was constructed in this study, and an indicator system for evaluating the SSCC of water diversion projects was built. The Dematel and 
Analytic Network Process (ANP) methods were used to clarify the mutual influence between evaluation indicators and identify key influencing factors and their importance, so as to improve the accuracy and validity of collaboration measurement. Finally, with the Middle Route of the South-to-North Water Diversion Project (SNWDP) as a case, the collaboration and evolution of the sustainable supply chain of the SNWDP were measured. This study aims to identify the "shortcomings" in the collaborative management of water diversion projects and provide an important reference for the operation and management of the SNWDP Middle Route and similar projects. This study also enriches the basic theoretical system on the SSC of water diversion projects and offers new ideas and scientific methods for the further research of SSCC mechanism.

\section{Definition of SSCC model for water diversion projects and its externalities}

The authors have consulted a large amount of literature before writing this paper, and many of them can provide important theoretical support for this paper. With reference to the research of Li, the authors constructed an SSCC model, as shown in Fig. 1 below (Li et al., 2021). Constructing water diversion projects is a national strategy to ensure the sustainable development of economy in different regions. Therefore, the operation and management of water diversion projects need to follow the economic, social and environmental benefits of a sustainable supply chain. 


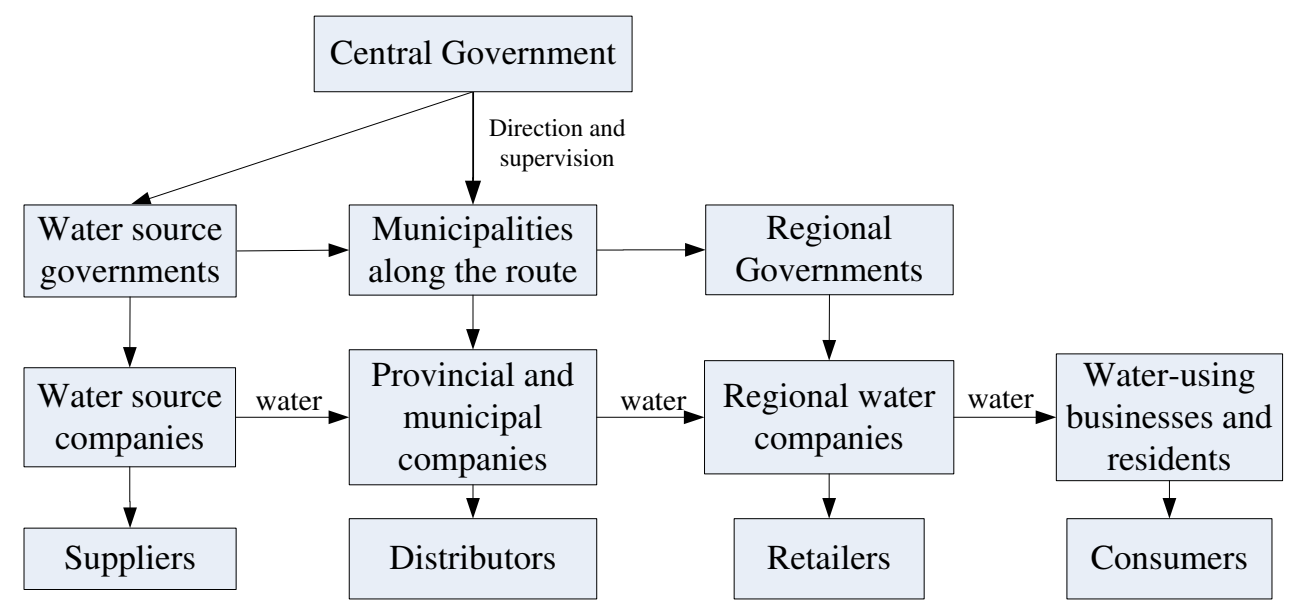

Economic, environmental and social triple aim sustainable supply chains

Fig. 1 Sustainable supply chain structure of water diversion projects

\section{SSCC model for water diversion projects}

The operation of conventional supply chain systems usually includes three important processes: information flow, cash flow, and material flow. Given the unique characteristics of the sustainable supply chain of water diversion projects, such as quasipublic welfare, intensive intersections with society, and the rich and colorful spiritual culture derived from the construction and operation of water diversion projects in China, like the spirit of the SNWDP, this paper constructs four flow subsystems including product flow, information flow, business flow, and knowledge flow.

\subsection{Construction of the indicator system}

The selection of indicators varies with the type of supply chain. In this paper, according to the operational characteristics of water diversion projects, indicators were classified as the product flow, information flow, business flow, and knowledge flow, and these flows were subdivided to obtain a more detailed SSCC evaluation indicator system, 
namely the order parameters for collaboration measurement.

\subsubsection{Product flow collaboration indicators}

The product flow of the water diversion projects' sustainable supply chain refers to the degree of coordination between the various links generated in the process of transporting water resources from the water source to the water users along the route, characterized by a long-term nature. When selecting the indicators, it is necessary to fully take into account the characteristics of water resources and the characteristics of product flow with water resources as products.

In their research on water diversion projects, many scholars have argued that the theoretical basis for the planning, operation and management of water diversion projects is the scientific allocation of water resources (Sun et al., 2003; Zhao et al., 2006). After field research and expert interviews, it was found that cross-regional water diversion projects have a defined regional water allocation scheme when it comes to water allocation. The total amount of water used in water diversion projects cannot serve as a direct basis for the success or failure of the project operation and scheduling, for the latter should be evaluated in combination of local water allocation indicators (You et al., 2018). Therefore, the pre-designed amount of water allocated and the actual amount of water distributed, namely the water quantity matching degree, can be selected as one of the indicators for evaluating the collaboration of the product flow. In addition, many scholars, in their studies of the SNWDP, have concluded that water quality is the key to the success of the project (Chen, 2016). Many water diversion projects not only meet the needs of production and living, generating economic benefits, 
but also greatly improve the ecological environment and water quality of the regions along the route, contributing much to the health of the people and the stability of society (Peng, 2018). Water users in each region are also consumers of the water diversion project's sustainable supply chain; thus, their satisfaction greatly affects the collaborative operation and management of the water diversion project. In addition, the daily activities of the residents along the water diversion route also influence the water quality, such as enterprises', households' and farmers' sewage discharge; therefore, the residents' willingness to protect the water diversion project also influence the collaborative management and operation of the project. In this paper, based on the research of $\mathrm{Hu}$ et al. (2017), Wang (2006) and Zhang (2016), the water quantity matching degree, water quality compliance rate, satisfaction with water use, and the willingness to protect were selected as the indicators for evaluating the collaboration of the product flow subsystem.

\subsubsection{Information flow collaboration indicators}

Information flow collaboration refers to the extent to which supply chain companies and individuals coordinate and cooperate in the collection, exchange, sharing and management of real-time data in a range of activities such as production, procurement, transportation, warehousing, and sales (Yao, 2018). Information collaboration can prevent the occurrence of the bullwhip effect and is also the basis of supply chain collaboration.

The operation and management of water diversion projects involve a balance between the water source and receiving area, the upstream and downstream, and the 
left and right banks, and also involve multi-objective resource management (Gao et al., 2018). The relationship of interests is complex and requires a high level of information collaboration along the route. Due to the particularity of the projects, it needs to collect diverse hydrological information for the project operation and management, such as multi-regional and multi-species information. All data that may affect the collaborative management and operation of the water diversion project need to be collected. The water diversion project is running continuously, generating a large amount of hydrological information, which needs to be collected and analyzed by operation managers in real time. Timely information acquisition by the operation management departments at each node contribute to the collaboration of the sustainable supply chain. At the same time, attention should be paid to the accuracy of the hydrological information collected. Incorrect information may lead to misjudgments by operation managers and sometimes cause serious consequences. In the field study, it was found that the information collected needs to be shared among the operation management departments. Therefore, based on Wang's research and taking into account the characteristics of water diversion projects (Wang, 2013), this study selects the comprehensiveness of hydrological information, timeliness of information transfer, accuracy of information, and willingness to share information to measure the collaboration of the information flow.

\subsubsection{Business flow collaboration indicators}

Business flow indicators mainly reflect the business management capacity during the operation and management of water diversion projects. Relevant management 
personnel expressed that in the actual operation and management of water diversion projects, they need to take immediate effective measures to deal with different risks brought about by different geographical and weather conditions of each region along the route. Therefore, the emergency response speed can be used to evaluate the business flow. The quantity of water diversion along the route is generally allocated by the water authority. The water surpluses in some areas may give rise to a water market where water resources are traded (Guo, 2017). The willingness to share water resources can also be used as an indicator to evaluate the operation and management of water diversion projects. The field interviews revealed that a large number of high technologies have been applied in the operation and management of current water diversion projects. Managers put forward that the innovation and application of high technologies should be further strengthened to cope with the complex geographical and climatic environments. Water diversion projects have delivered large amounts of clean water to the receiving areas, solving their water shortages. However, the water source areas pay a huge price to ensure a stable long-term supply of clean water resources (Xie, 2017). For example, due to the Danjiangkou Dam heightening project in the water source area of the SNWDP Middle Route, 345,000 people had to relocate. Therefore, it is particularly important to establish a compensation mechanism that satisfies the public while taking into account the coordinated development of ecology and economy and social stability (Li et al., 2021). In addition, the reasonableness of the water price and the water users' satisfaction are related to the feasibility of the water diversion project and also directly affect the subsequent benefits of the project and its continued operation 
(Qian et al., 2017). Therefore, in this study, the application of high technologies, satisfaction with the compensation mechanism, and satisfaction with the water price were selected as the order parameters of the business flow subsystem.

\subsubsection{Knowledge flow collaboration indicators}

China has built many water diversion projects, which have long formed a body of knowledge. With the continuous construction, operation and management of the projects, they have developed their own unique spirit and culture. For example, the SNWDP has developed a unique spirit of "national planning, people first, innovation and aspiration for perfection, dedication and commitment" (Sun et al., 2003), and accordingly formed an exclusive cultural system. The understanding of the project culture by managers and stakeholders along the route contributes to the benefits of the project and its continued operation. Through field research and interviews, it was found that many of the middle management of the SNWDP Middle Route were promoted from the technical backbone of the project during its construction, and they lacked the ability to coordinate complex interests. Furthermore, managers have less time to spend in learning relevant knowledge, and fewer learning and exchange meetings are held in regions along the route. This phenomenon is not conducive to the managers' learning and updating of relevant professional knowledge, nor is it conducive to the exchange and sharing of knowledge among peers. The consistency of development strategies in the regions along the route is helpful for the sustainable operation of the project and the immediate response to risks. Therefore, in this paper, the understanding of the project spirit and culture, the time spent by managers in learning, the number of training 
meetings held, the willingness to share knowledge, and the consistency of development strategies were selected as the order parameters of the knowledge flow subsystem.

The indicators for evaluating the SSCC of water diversion projects are listed in

\section{Table 1 below.}

Table 1 Evaluation indicator system for the sustainable supply chain collaboration of water diversion projects

\begin{tabular}{ll}
\hline Subsystem & Order parameters \\
\hline
\end{tabular}

Water quantity matching degree A1

Satisfaction with water use A2

Product flow S1

Water quality compliance rate $\mathrm{A} 3$

Willingness to protect A4

Comprehensiveness of information collected B1

Timeliness of information sharing B2

Sustainable supply

chain collaboration of

water diversion

projects
Information flow S2

$$
\text { Accuracy of information B3 }
$$

Degree of information sharing B4

Emergency response speed $\mathrm{C} 1$

Willingness to share water resources $\mathrm{C} 2$

Application of high technologies C3

Satisfaction with compensation mechanism C4

Satisfaction with water pricing C5

Understanding of spiritual culture D1

Knowledge flow S4 


\subsection{Determination of the relationship between collaboration evaluation indicators} and their weights based on Dematel and ANP

The combined Dematel-ANP method was used in this study to analyze the SSCC indicator system of water diversion projects.

(1) The Dematel method can sort out the complex relations among indicators, calculate the centrality and causality of each indicator, and build a cause-effect diagram to clarify the internal connection of each indicator and its role in the overall collaboration capacity. (2) The ANP method is improved based on the Analytical Hierarchy Process (AHP) method. It emphasizes the influence and correlation between the indicators in the whole collaboration, which conforms to the SSCC evaluation indicators of water diversion projects. (3) The correlation of indicators obtained through the Dematel method and the weights of indicators obtained through the ANP method are combined to obtain a reasonable and objective indicator system.

This paper summarizes the research of relevant scholars and constructs a comprehensive evaluation model based on Dematel-ANP, as shown in Fig. 2 below (Duan et al., 2019; Wang et al., 2021). 


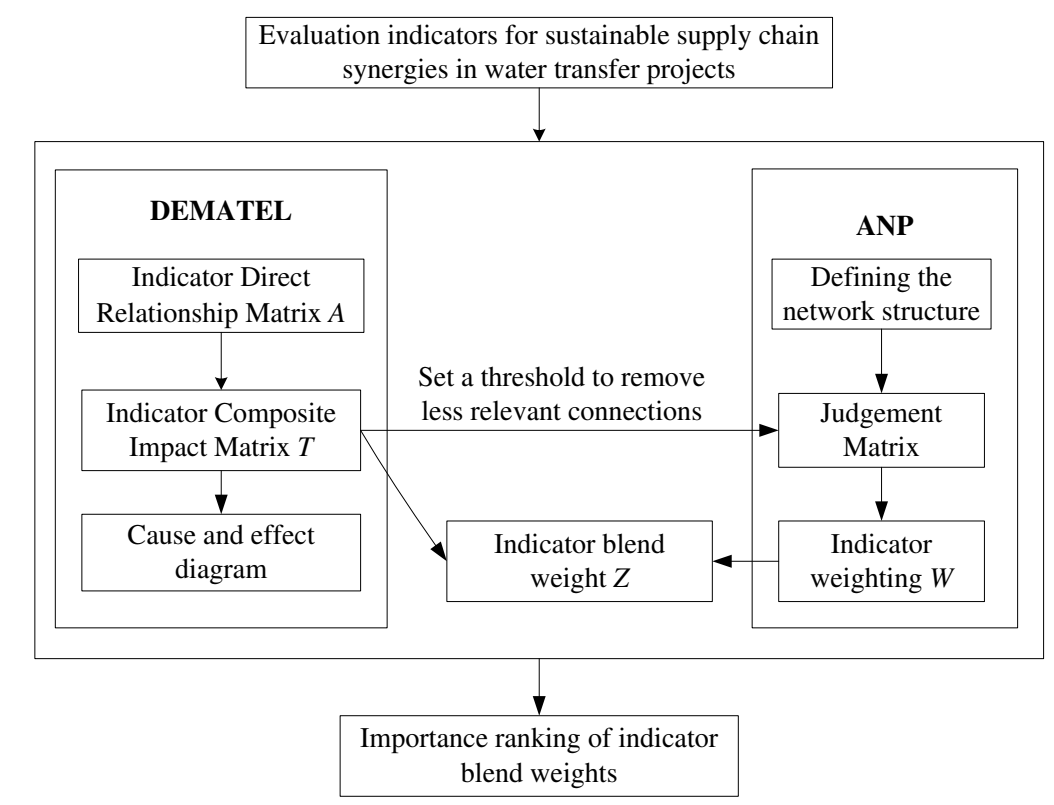

Fig. 2 Evaluation model of sustainable supply chain collaboration capacity indicators for water diversion projects

\subsubsection{Determination of the cause-effect diagram based on the Dematel method}

The Dematel method can simplify the relationship between factors within the system into an easy-to-understand relational structure (GABUS et al., 2008). It uses the graph theory and matrix tools to analyze indicators and calculate the centrality and causality through the logical relationship within the indicators (Tang et al., 2013).

(1) Based on the SSCC indicator system constructed for water diversion projects, the direct influence between subsystems was estimated, and a direct-relation matrix $A$ was thus formed. The 5-level scaling method was used to measure the degree of influence between factors. 1 meant no influence, 2 meant little influence, 3 meant general influence, 4 meant strong influence, and 5 meant very strong influence.

(2) The direct-relation matrix $A$ was normalized to obtain the matrix $X$. The DEMATEL related procedure was used with the aid of MATLAB software to figure out the total relation matrix $T$ in accordance with the following equation. 


$$
\begin{gathered}
X=\lambda \times A \\
\lambda=\min \left(\frac{1}{1 \leq i \leq n \sum_{j=1}^{n}\left|a_{i j}\right|}, \frac{1}{1 \leq j \leq n \sum_{i=1}^{n}\left|a_{i j}\right|}\right) \\
T=\sum_{k=1}^{+\infty} X^{k}=X(I-X)^{-1}
\end{gathered}
$$

(3) The degree of influence $D$, the degree of being influenced $R$, the centrality $D+R$, and the causality $D-R$ were calculated by the following equation, respectively. The centrality of an indicator indicates the position and role of the indicator in the whole complex system. If the causality is greater than 0 , it represents a cause indicator that may affect other indicators; if the causality is smaller than 0 , it represents a result indicator that may be affected by other indicators.

$$
T=\left(t_{i j}\right)_{n \times n}, i, j \in\{1,2, \Lambda, n\} ; D=\sum_{j=1}^{n} t_{i j} ; R=\sum_{i=1}^{n} t_{i j}
$$

(4) The cause-effect diagram drawn based on the values of centrality $D+R$ and causality $D-R$ shows the causality between the indicators.

\subsubsection{Determination of the indicator weights based on the ANP method}

The ANP method is a decision-making method developed based on the AHP. It was first proposed by Satty (2004) who believed that indicators are not independent of each other, but are interdependent and affect each other. It is more applicable to the interrelated internal subsystems, closer to the objective reality, and more scientific and accurate than the AHP. Its calculation steps are as follows.

(1) Construct a network structure between indicators using SD software based on 
the cause-effect diagram.

(2) Construct a supermatrix. In the network layer, all elements were compared in pairs in terms of importance under the control layer criterion to build a supermatrix and then to obtain the feature vectors (Duan et al., 2019); finally, the matrix was obtained as follows.

$$
W_{i j}\left|\begin{array}{cccc}
W_{i 1}^{\left(j_{1}\right)} & W_{i 1}^{\left(j_{2}\right)} & \Lambda & W_{i 1}^{\left(j_{n}\right)} \\
W_{i 2}^{\left(j_{1}\right)} & W_{i 2}^{\left(j_{2}\right)} & \Lambda & W_{i 2}^{\left(j_{n}\right)} \\
\mathrm{M} & \mathrm{M} & \mathrm{M} & \mathrm{M} \\
W_{i n}^{\left(j_{1}\right)} & W_{i n}^{\left(j_{2}\right)} & \Lambda & W_{i n}^{\left(j_{n}\right)}
\end{array}\right|
$$

(3) Construct a weighted supermatrix. The influence of other layers were not taken into account in the said step to construct the supermatrix, so priority vectors among the indicators were combined to form the supermatrix $W$, and then the supermatrix $W$ was normalized to obtain the weighted supermatrix.

(4) Limit supermatrix. The limit of the weighted supermatrix was computed to better reflect the correlation between indicators.

$$
W^{*}={ }_{k \rightarrow+\infty} W^{k}
$$

\subsubsection{Determination of the blend weights}

Among the said indicators, one may not have a high weight, but its correlation is high. Improving the ability of such indicators can increase the whole collaboration capacity. Dematel was used to derive the correlation among all indicators, and ANP was used to derive the weight of each indicator. The blend weights of the collaboration evaluation indicators were calculated using the following formula. The blend weights can directly 
reflect the weight of each collaboration indicator in the system and its importance.

$$
Z=W+T \times W=(I+T) W
$$

where $Z$ is the blend weight; $W$ is the weight of indicators; $T$ is the total relation matrix of indicators; and $I$ is the unit matrix.

\subsection{System collaboration model}

The relationship between the subsystems and the whole system is not a simple linear sum. Therefore, when constructing the evaluation model for the SSCC of water diversion projects, it needed to evaluate the orderliness of the "four flow" subsystems first; then, the difference and dispersion between the subsystems were calculated; and finally, the collaboration of the whole supply chain of water diversion projects was obtained through the mathematical model.

The evaluation model included the power function, the subsystem collaboration function, the system collaboration function, the ordered dispersion of subsystems, and the collaboration of the entire sustainable supply chain of the water diversion project. Their definitions and formulas are as follows (Yin, 2018).

\subsubsection{Power function}

The change in the order parameters of the system affects the orderliness of the system. As the order parameter increases, the orderliness of the system increases accordingly, which is called positive collaboration; on the contrary, as the order parameter increases, the orderliness of the system decreases, which is called negative collaboration. Assume that the synergistic effect of the order parameter on the system is $E C$, which takes the 
value between 0 and 1 . When $E C=1$, the synergistic effect is the largest; when $E C=0$, the synergistic effect is the smallest.

Assume that any of the four flow subsystems of the sustainable supply chain of water diversion projects is $S_{j}(j=1,2,3,4)$, and meanwhile assume that $S_{j}$ contains $n$ order parameters $\left[e_{j i}=\left(e_{j 1}, e_{j 2}, \ldots, e_{j n}\right)\right]$ in its development process, $n \geq 1, \beta_{j i} \leq e_{j i} \leq \alpha_{j i}$, $i \square[1, n] . \alpha$ and $\beta$ are upper and lower limits of the stable $e_{j i}$ in the subsystem. In the subsystem $S_{j}$, assume that the first $m(m \leq n)$ variables $\left(e_{j 1}, e_{j 2}, \ldots, e_{j m}\right)$ take the larger value, then the orderliness of the sustainable supply chain is higher; assume that the $n$ $m$ variables $\left(e_{j(m+1)}, e_{j(m+2)}, \ldots, e_{j n}\right)$ take the larger value, then the orderliness of the system is lower. Thus, the effect of the order parameter $e_{j i}$ on the orderliness of the sustainable supply chain subsystem $S_{j}$ of the water diversion project is defined as follows:

$$
E C_{j}\left(e_{j i}\right)=\left\{\begin{array}{l}
\frac{e_{j i}-\beta_{j i}}{\alpha_{j i}-\beta_{j i}}, i \in[1, m] \text { (Positive efficacy) } \\
\frac{\alpha_{j i}-e_{j i}}{\alpha_{j i}-\beta_{j i}}, i \in[m+1, n] \text { (Negative efficacy) }
\end{array}\right.
$$

\subsubsection{Subsystem collaboration function}

To compute the subsystem orderliness of the sustainable supply chain of water diversion projects, assuming that the order parameter weight of each subsystem is wi, then $w_{i} \geq 0, \sum_{i=1}^{n} w_{i}=1$. The subsystem orderliness not only depends on the specific value of each order parameter component, but also has an inextricable link with the combination form between them. With reference to the subsystem collaboration calculation method proposed by Wang et al.(2019), this paper employs the linear 
weighted sum to calculate the subsystem collaboration.

$$
O C_{j}\left(S_{j}\right)=\sum_{i=1}^{n} w_{i} E_{c j}\left(e_{j i}\right)(i=1,2, \Lambda, n)
$$

Since $E C_{j}\left(e_{j i}\right) \in[0,1]$, the orderliness of the supply chain subsystem $O C_{j}\left(S_{j}\right)$ is also between 0 and 1 . The subsystem $S_{j}$ has the highest orderliness when $O C_{j}\left(S_{j}\right)$ takes 1 , and has the lowest orderliness when $O C_{j}\left(S_{j}\right)$ takes 0 .

\subsubsection{Subsystem collaboration function}

Based on relevant research findings, the system collaboration of the sustainable supply chain of water diversion projects in this paper was defined as the overall system orderliness expressed by the combination of the orderliness of each subsystem. The orderliness of each subsystem may affect the overall collaboration of the system. The SSCC of water diversion projects was defined as a linear weighted sum of the orderliness of each subsystem. Assuming that the weight of the subsystem is $y_{j}$, then $y_{j} \geq 0, \sum_{j=1}^{n} y_{j}=1$, the specific formula is as follows:

$$
C C=\sum_{j=1}^{n} y_{j} O C_{j}\left(S_{j}\right)(j=1,2,3,4)
$$

where $C C$ is the collaboration of the sustainable supply chain of water diversion projects, and $O C_{j}\left(S_{j}\right)$ is the orderliness of the sustainable supply chain subsystems of water diversion projects.

\subsubsection{Ordered dispersion of subsystems}

As the four subsystems have different attributes, the differences and interactions between these subsystems may affect the overall collaboration of the sustainable supply 
chain of water diversion projects. The difference between subsystems needs to be calculated first before calculating the overall collaboration. In this paper, following the previous solutions to such problem, the standard deviation rate was used to reflect the degree of dispersion, namely the difference between each subsystem and the average orderliness.

$$
D=\frac{\delta}{C C\left(S_{j}\right)}=\frac{\sqrt{\sum_{j=1}^{n} \frac{\left[O C_{j}\left(S_{j}\right)-C C\left(S_{j}\right)\right]^{2}}{n-1}}}{C C\left(S_{j}\right)}
$$

where $\delta$ is the standard deviation of the orderliness of each subsystem of the sustainable supply chain of water diversion projects, and $C C\left(S_{j}\right)$ is the collaboration of the sustainable supply chain system of water diversion projects.

\subsubsection{Collaboration of the sustainable supply chain of water diversion projects}

The collaboration of the sustainable supply chain of water diversion projects and the matching degree between each subsystem are not independent, but influence each other. When one of the two changes, the effect of the other on the SSCC of water diversion projects will also change accordingly. The SSCC of water diversion projects is determined based on the mutual influence of the two. Given this, the formula for calculating the collaboration in this paper is as follows.

$$
C I=C C \cdot(1-D)=\sqrt[n]{\prod_{j=1}^{n} y_{j} O C_{j}\left(S_{j}\right)} \cdot\left[1-\frac{\sqrt{\sum_{j=1}^{n} \frac{\left[O C_{j}\left(S_{j}\right)-C C\left(S_{j}\right)\right]^{2}}{n-1}}}{C C\left(S_{j}\right)}\right]
$$




\section{Empirical analysis of the SNWDP Middle Route}

\subsection{Calculations of weights}

To ensure scientific and accurate evaluation results, a total of nine water diversion project experts and managers along the SNWDP were invited to judge the mutual influence between indicators and score them using the Delphi method. Thus, a directrelation matrix was constructed, and then the total relation matrix was obtained. The causality threshold was set to 0.66 according to the experts' suggestion. Based on the total relation matrix, the causality and centrality were derived as shown in Table 2 below.

Table 2 Causality and Centrality

\begin{tabular}{|c|c|c|c|c|c|c|c|}
\hline & \multirow{3}{*}{ S1 } & \multirow{3}{*}{$\mathrm{S} 2$} & \multirow{3}{*}{ S3 } & \multirow{3}{*}{ S4 } & \multirow[t]{2}{*}{ Degree of } & \multirow{3}{*}{ Centrality } & \multirow{3}{*}{ Causality } \\
\hline & & & & & & & \\
\hline & & & & & influence & & \\
\hline $\mathrm{S} 1$ & 0.0000 & 0.7756 & 0.8260 & 0.7649 & 2.3665 & 4.0915 & 0.6415 \\
\hline $\mathrm{S} 2$ & 0.9665 & 0.7509 & 1.0739 & 1.0508 & 3.8421 & 6.8491 & 0.8351 \\
\hline $\mathrm{S} 3$ & 0.7585 & 0.7700 & 0.0000 & 0.8002 & 2.3287 & 4.9638 & -0.3064 \\
\hline $\mathrm{S} 4$ & 0.0000 & 0.7105 & 0.7352 & 0.0000 & 1.4457 & 4.0616 & -1.1702 \\
\hline \multicolumn{8}{|c|}{ Degree of being } \\
\hline & 1.7250 & 3.0070 & 2.6351 & 2.6159 & & & \\
\hline influenc & & & & & & & \\
\hline
\end{tabular}

Based on the said results, the ANP network of the SSCC evaluation system of water diversion projects was drawn, as shown in Fig. 3 below, containing a target layer and a network layer. The internal relationship in the network layer was determined by the causal relation between the first-tier indicators. 


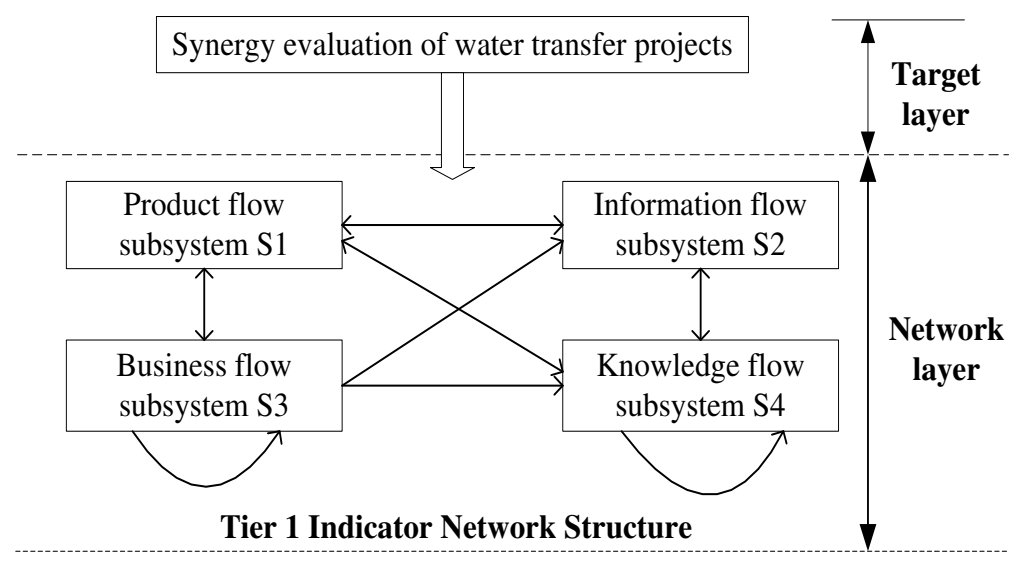

Fig. 3 ANP network for evaluating the sustainable supply chain collaboration of water diversion projects

Based on the final influence matrix and the cause-effect diagram, the paired judgment matrix questionnaire filled in by the water diversion project experts was input into the SD software to test its consistency. When the consistency coefficient was less than 0.1 , the judgment matrix would be accepted to form a supermatrix. SD software also generated weights, as shown in Fig. 4 below.

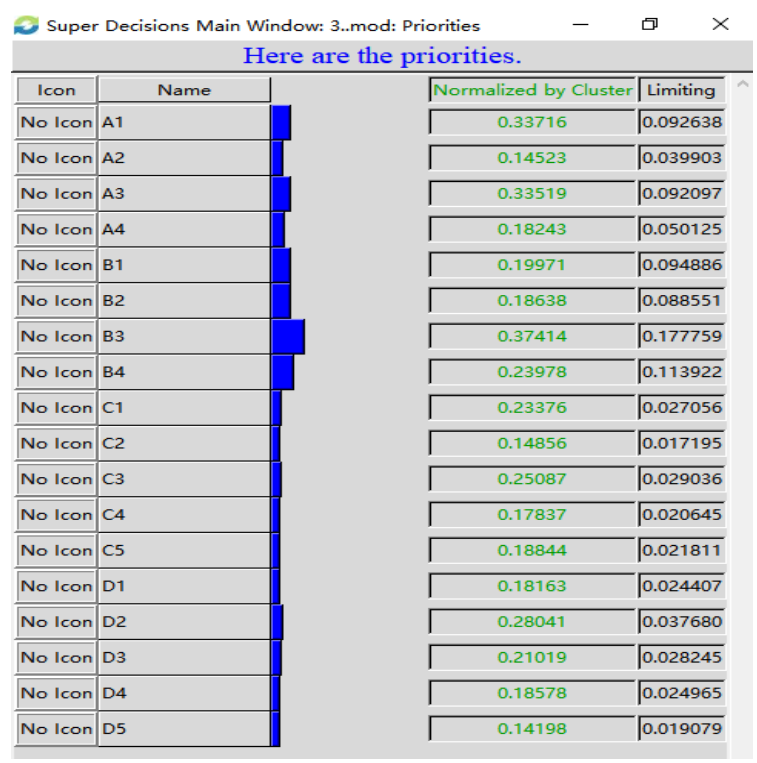

Fig. 4 SD software results

The results are presented in a table, as shown in Table 3 below.

Table 3 Weight of indicators for evaluating the sustainable supply chain of water diversion projects 


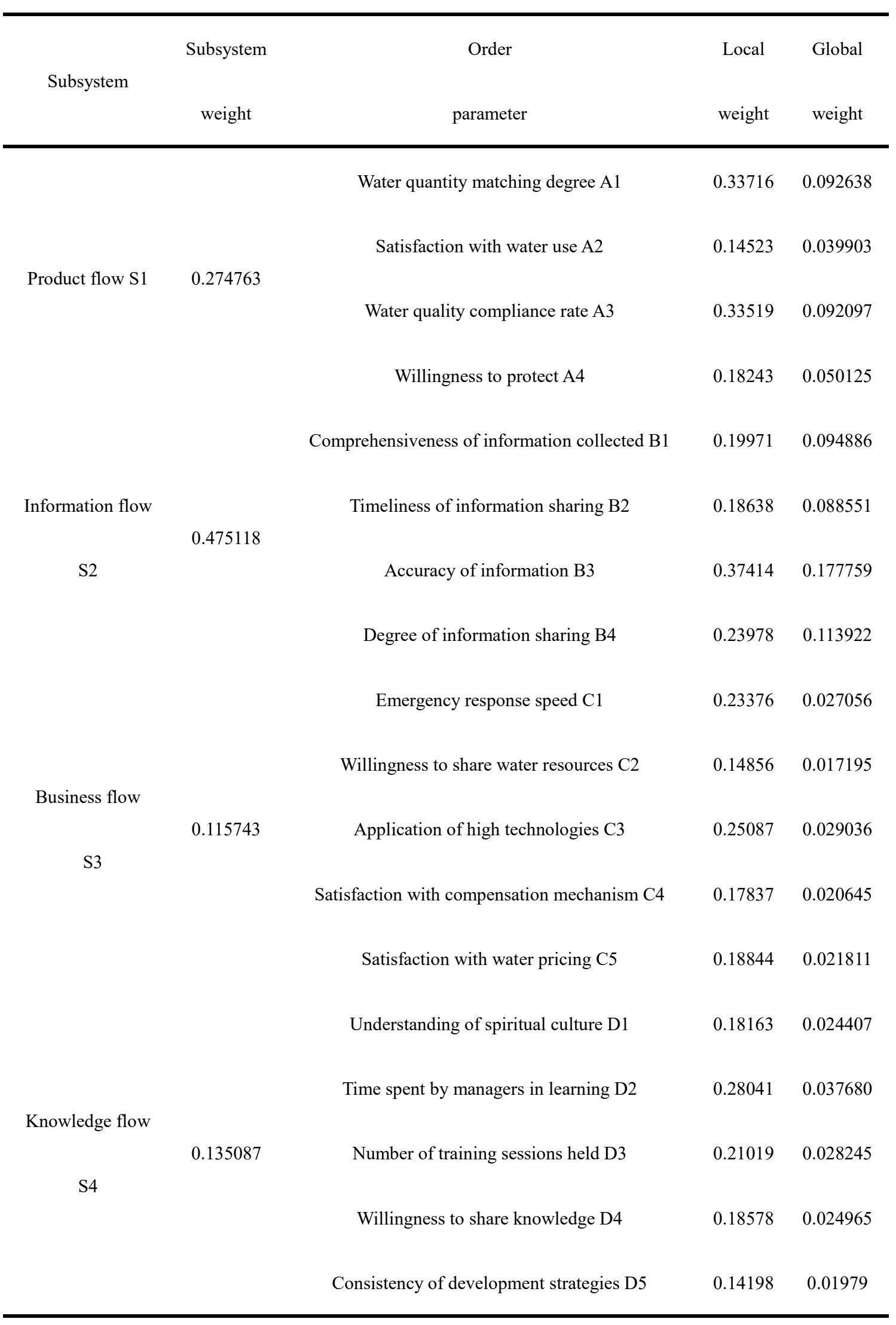

\subsection{Measurement of collaboration}

The original data was obtained by means of questionnaires and interviews. First, the 
original questionnaire was set up according to the evaluation indicators of collaborative management, and the indicators were mainly quantitative. Specialized data was obtained through interviews with managers along the SNWDP. In order to accurately reflect the current management status of the SNWDP, the questionnaire was designed with reference to a large amount of literature related to management, collaborative management and sustainable supply chain management of the SNWDP (He, 2013; Wang, 2017; Wang et al., 2019; Yin et al., 2020). A total of 22 questions were listed in the questionnaire based on 18 order parameters, with product flow, information flow, business flow, and knowledge flow as the primary indicators.

The preliminary questionnaire was distributed to relevant experts. Based on their feedback, it was revised repeatedly. The questions were set up in an easy-to-understand manner, taking into account the specific management issues and the characteristics of the people involved. The formal questionnaire was eventually developed under the guidance of the supervisor.

\subsubsection{Data acquisition}

The SNWDP Middle Route was selected as a case, and the samples were collected from the water source areas, Henan, Hebei, Tianjin, and Beijing along the route. To ensure that the samples were representative, at least 25 questionnaires were distributed in the water source areas and other areas along the route. To ensure that the questionnaires achieved the pre-designed effect, five different links were set up for different areas using the Wenjuanxing software. A total of 164 questionnaires were collected in one month. Excluding three invalid questionnaires, 161 questionnaires were retained. The 
validity of the questionnaire reached 98.17. In addition, we interviewed seven managers along the route. The valid sample size in this study was close to a medium-sized sample (200) and was five times greater than the number of questions set in the questionnaire (Kaplan et al., 1999). Thus, it could be considered that the empirical analysis in this study was reliable.

\subsubsection{Questionnaire results}

The results of the questionnaires are collated as shown in Table 4 below.

Table 4 Collaboration Measurement Results of the South-to-North Water Diversion Project Middle Route

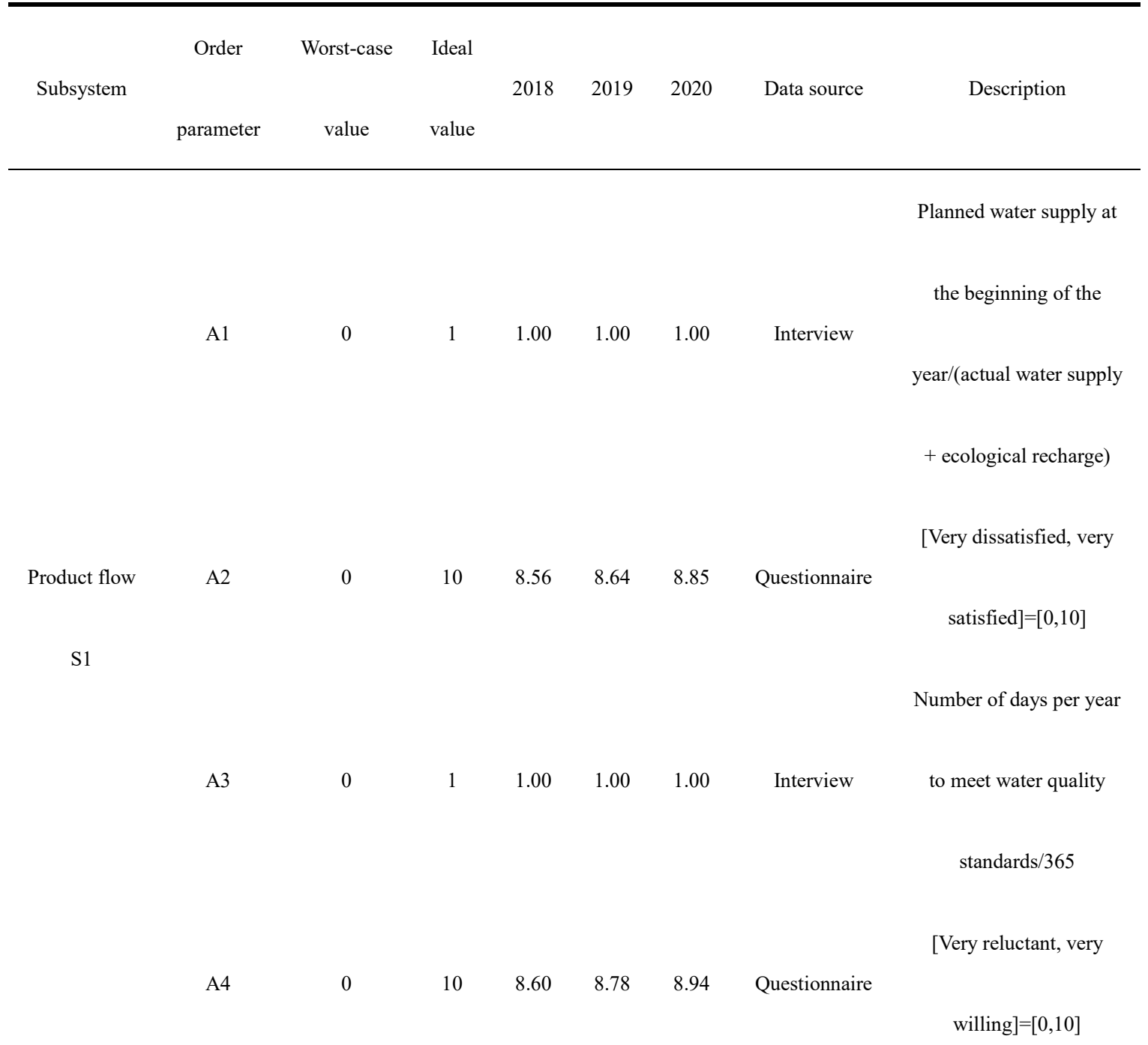




\begin{tabular}{|c|c|c|c|c|c|c|c|c|}
\hline & & & & & & & & [Very incomplete, very \\
\hline & $\mathrm{B} 1$ & 0 & 10 & 7.62 & 8.00 & 8.31 & Questionnaire & \\
\hline & & & & & & & & complete $]=[0,10]$ \\
\hline & & & & & & & & Real-time information \\
\hline & B2 & 0 & 1 & 1.00 & 1.00 & 1.00 & Interview & \\
\hline Information & & & & & & & & sharing \\
\hline flow S2 & & & & & & & & [Very accurate, very \\
\hline & B3 & 0 & 10 & 9.62 & 9.85 & 10.0 & Questionnaire & \\
\hline & & & & & & & & inaccurate $]=[0,10]$ \\
\hline & & & & & & & & Real-time information \\
\hline & B4 & 0 & 1 & 1.00 & 1.00 & 1.00 & Interview & \\
\hline & & & & & & & & sharing \\
\hline & & & & & & & & [Very untimely, very \\
\hline & $\mathrm{C} 1$ & 0 & 10 & 7.92 & 7.85 & 8.00 & Questionnaire & \\
\hline & & & & & & & & timely $]=[0,10]$ \\
\hline & & & & & & & & [Very reluctant, very \\
\hline & $\mathrm{C} 2$ & 0 & 10 & 7.38 & 7.92 & 8.15 & Questionnaire & \\
\hline & & & & & & & & willing $]=[0,10]$ \\
\hline Business flow & & & & & & & & Applied type/applied type \\
\hline & $\mathrm{C} 3$ & 0 & 1 & 0.66 & 0.68 & 0.65 & Interview & \\
\hline S3 & & & & & & & & + desired type \\
\hline & & & & & & & & [Very dissatisfied, very \\
\hline & $\mathrm{C} 4$ & 0 & 10 & 8.36 & 8.43 & 8.63 & Questionnaire & \\
\hline & & & & & & & & satisfied $]=[0,10]$ \\
\hline & & & & & & & & [Very dissatisfied, very \\
\hline & $\mathrm{C} 5$ & 0 & 10 & 8.30 & 8.46 & 8.54 & Questionnaire & \\
\hline & & & & & & & & satisfied $]=[0,10]$ \\
\hline & & & & & & & & [Very unfamiliar, very \\
\hline & D1 & 0 & 10 & 7.45 & 7.44 & 7.96 & Questionnaire & \\
\hline Knowledge & & & & & & & & familiar $]=[0,10]$ \\
\hline flow S4 & & & & & & & & Average learning time of \\
\hline & D2 & 0.5 & 3 & 1.5 & 1.5 & 1.5 & Interview & \\
\hline
\end{tabular}


D3

\subsubsection{Reliability and validity tests}

The reliability and validity of data used in this paper were tested using SPSS 22.0 statistical software. The results are shown in Table 5 below.

Table 5 Reliability and validity of the questionnaire

\begin{tabular}{|c|c|c|}
\hline Questionnaire indicators & Cronbach's Alpha & KMO value \\
\hline Satisfaction with water use A2 & 0.966 & 0.69 \\
\hline Willingness to protect A4 & 0.949 & 0.656 \\
\hline Comprehensiveness of information collected B1 & 0.987 & 0.639 \\
\hline Accuracy of information B3 & 0.986 & 0.778 \\
\hline Emergency response speed $\mathrm{C} 1$ & 0.983 & 0.667 \\
\hline Willingness to share water resources $\mathrm{C} 2$ & 0.992 & 0.769 \\
\hline Satisfaction with compensation mechanism C4 & 0.873 & 0.584 \\
\hline Satisfaction with water pricing C5 & 0.974 & 0.745 \\
\hline
\end{tabular}


Understanding of spiritual culture D1

Willingness to share knowledge D4

Consistency of development strategies D5
0.988

0.638

0.99

0.655

Results in the above table showed that the questionnaire passed the reliability and validity tests and could enter the next step of collaboration analysis.

\subsubsection{Measurement of the collaboration of the SNWDP Middle Route}

The calculation results are shown in Table 6 below. The development trend of the orderliness of each subsystem is shown in Fig. 5.

Table 6 Orderliness of sustainable supply chain subsystems, 2018-2020

\begin{tabular}{cccc}
\hline Subsystem & 2018 & 2019 & 2020 \\
\hline Product flow & 0.954 & 0.958 & 0.962 \\
Information flow & 0.937 & 0.956 & 0.966 \\
Business flow & 0.766 & 0.783 & 0.785 \\
Knowledge flow & 0.555 & 0.553 & 0.565 \\
\hline
\end{tabular}




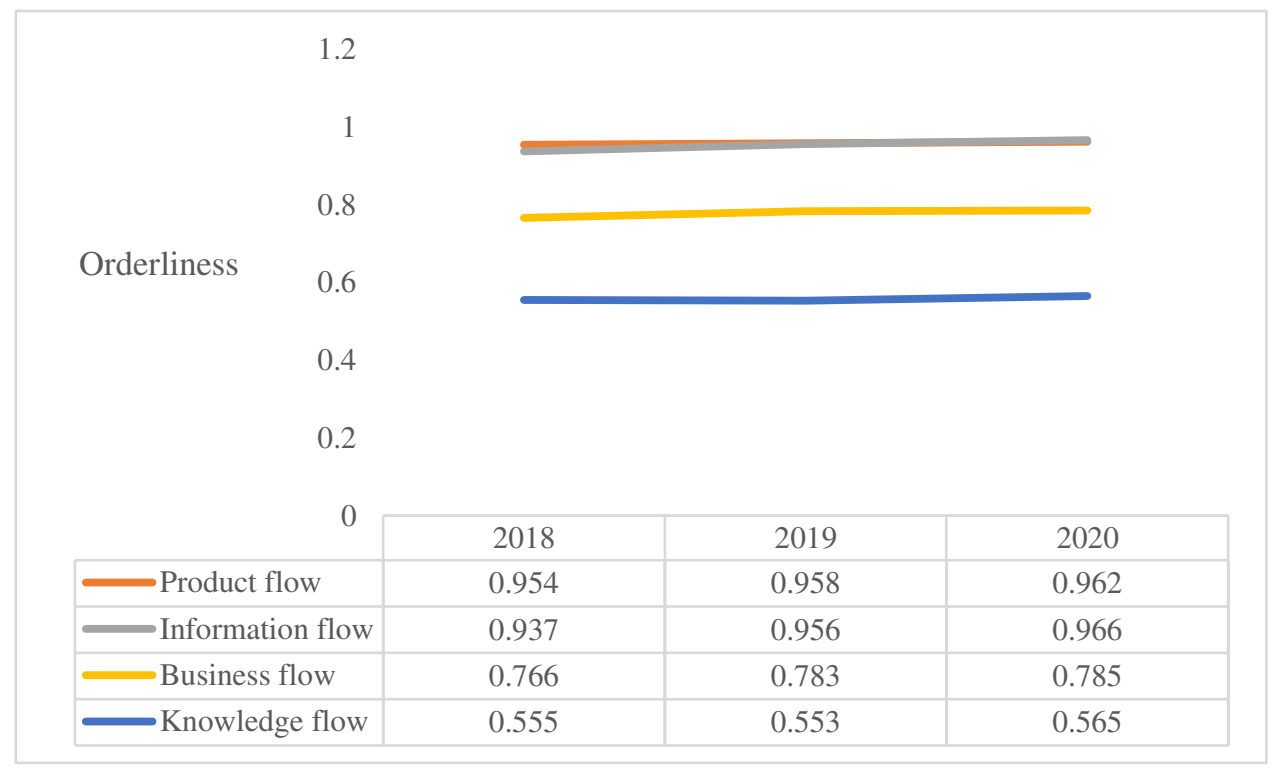

Fig. 5 Orderliness trend of each subsystem

The results of the supply chain collaboration capability, four flows' orderliness and supply chain collaboration are shown in Table 7 below. The development trend of SSCC is shown in Fig. 6 below.

Table 7 Sustainable supply chain collaboration parameters (2018-2020)

\begin{tabular}{cccc}
\hline Parameter & 2018 & 2019 & 2020 \\
\hline Supply chain collaboration capability & 0.871 & 0.883 & 0.890 \\
Ordered dispersion of the subsystem & 0.232 & 0.236 \\
Supply chain collaboration & 0.669 & 0.675 & 0.684 \\
\hline
\end{tabular}




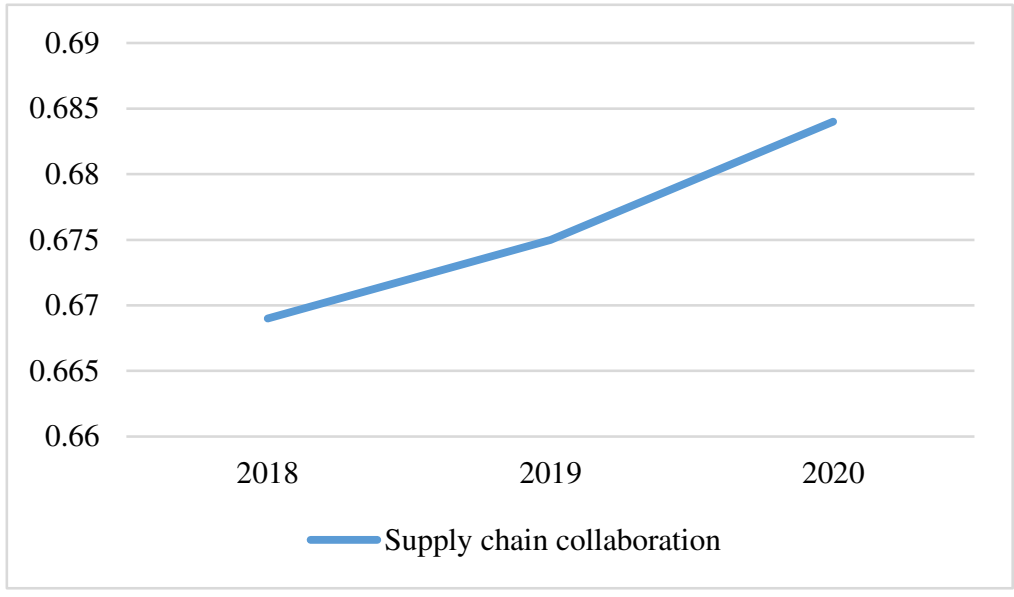

Fig. 6 Trends of the sustainable supply chain collaboration

\subsubsection{Evaluation of results}

As can be seen in Fig. 5, The high orderliness of the product flow indicated that a great deal of attention had been paid to water quantity and quality in the collaborative operation and management of the SNWDP. Water users along the route had a high satisfaction with the water and a high awareness of environmental protection. The orderliness of the information flow also reached a high level and even surpassed the orderliness of the product flow in 2020. This meant that the orderliness of the information flow subsystem was gradually increasing with the widespread use of modern information technology in the operation and management of the SNWDP. The sustainable supply chain of the SNWDP became more collaborative overall.

As can be seen in Fig. 6, the collaboration of the sustainable supply chain of the SNWDP is on the rise. Interviews with officials along the SNWDP showed that a large number of high technologies have been used in the current operation and management of the SNWDP, meeting daily information exchange and real-time sharing along the route. In recent years, there are few changes in the application of smart technologies, 
and real-time monitoring of water quality and quantity has been achieved at each section gate.

For all that, technologies should be upgraded to cope with the complex geographical and climatic environments. Operation managers hoped that in the future, real-time monitoring and risk identification and early warning would be both realized. For example, the use of underwater robots and drones under complex hydrological conditions and harsh weather conditions may enable rapid and automatic identification of defects in project maintenance and construction. The SNWDP Administration expected active cooperation from all branch offices to achieve network-wide, timesensitive and dead zone-free supervision. In the knowledge flow subsystem, interviews revealed that current managers spent less time studying and few exchange meetings were held along the route. This affected the orderliness of the knowledge flow subsystem and the collaboration of the whole supply chain. Therefore, managers proposed that more management exchange and training meetings should be organized along the route. This would enable better operation and management of the SNWDP Middle Route, so that the project can create more economic, environmental and social benefits.

\section{Shortcomings and prospects}

In this study, the synergetics theory and collaboration measurement model were used to analyze the sustainable supply chain system of water diversion projects. However, due to few findings available for reference, this paper has some shortcomings that need to be improved in the subsequent studies. 
(1) We read relevant literature and found that the previous studies about the supply chain collaboration involved a wide time span, while in this study, we only collected three-year data of the SNWDP Middle Route from 2018 to 2020. The time span and the research object need to be increased during the subsequent studies.

(2) Although in this study, we constructed four subsystems of product flow, information flow, business flow, and knowledge flow, we didn't make further investigations into the mechanism by which the four subsystems affect the SSCC, and nor analyze the dynamic evolution of the entire sustainable supply chain system to achieve collaboration.

(3) The criterion for the selection of indicators in the empirical part needs to be improved. We consulted a lot of relevant literature and data when selecting the order parameter indicators, but given the data availability and our limited ability to handle data, the final indicator system still needs to be improved. In the future research, more attention should be paid to the standardization of the indicator system and its intrinsic meaning.

Declaration of Competing Interest The authors declare that they have no known competing financial interests or personal relationships that could have appeared to influence the work reported in this paper.

Acknowledgments This study was supported financially by National Natural Science Foundation of China (grant numbers: 71974056); Science and technology innovation talent support plan of colleges and universities in Henan Province (grant numbers: 2021-CX-005); Henan Science and Technology Think Tank Research Project (grant numbers: HNKJZK-2022-04B); China Scholarship Council (2020) 28 (File NO. 202008410271)

Author Contributions All authors contributed to the study conception and design. Material preparation, data collection and analysis were performed by M Liu, WW Ding 
and YL Lu. The first draft of the manuscript was written by M Liu and all authors commented on previous versions of the manuscript. All authors read and approved the final manuscript.

\section{References}

Andrew Campbell, Kathleen Sommers Luchs (2003) Translated by Ren Tonghai,Long Dawei. Strategic Synergy (2nd ed.) [M]. Beijing: Machinery Industry Press.

Berkes F (2017) Environmental governance for the anthropocene? Social-ecological systems, resilience, and collaborative learning. Sustainability 9 (7), 1232.

Chen W (2016) The key to the success or failure of the South-North Water Transfer Project: water quality or water quantity. China Soft Science(7), 9.

Chen ZS, Wang HM (2012) Optimization and coordination of south-to-north water diversion supply chain with strategic customer behavior. Water Science and Engineering, 5(4), 14.

Chen Z, H Wang, X Qi (2013) Pricing and water resource allocation scheme for the South-to-North water diversion project in China. Water Resources Management 27 (5): 1457-1472.

Duan YL, Huang L, Zhang XQ (2019) Research on the evaluation of innovation capability of strategic emerging industries based on dematel-anp. Science and Technology Management Research, 39(13), 11.

GABUS A, FONTELA E (2008) World problems, an invitation to further thought within the framework of DEMATEL.[J].Switzerland Geneva:Battelle Geneva Research, 5(3):160-168.

Gao SP, Jiang H, Zhang X (2020) Research on the development trend of regional science and technology resources synergy and ecological governance mechanism. Science and Technology Progress and Countermeasures, 37(17), 10.

Gao YY, Yao JW, Chen GF et al (2018) Current status and outlook of water transfer 
projects in China. China Water Resources, 000(004), 49-51.

German Haken (1984) Synergetics: Introduction to Non-Equilibrium Phase

Transitions and Self-Organization in Physics, Chemistry and Biology. Atomic Energy Press.

Guo H (2017) Exploring the construction of water rights trading market in the middle line of South-North water transfer[J]. Water Resources Development Research, 17(06):16-21+27.

Gu WQ, Shao DG, Tan XZ et al (2017) Simulation and optimization of multireservoir operation in inter-basin water transfer system. Water Resources Management,31(11):3401-3412.

Haken H (2004) Synergetics: Introduction and Advanced Topics[M]. Springer Berlin Heidelberg.

He CH, Zhou XC (2013) Drivers and constraints of sustainable supply chain management. Hunan Social Science(1), 5.

Hu YY, Tang PP (2017) Research on the performance evaluation of water ecological civilization construction in the Han River water source of the South-North Water Diversion Central Project. Ecological Economy, 33(2), 4.

Kaplan D, Ferguson AJ (1999) On the utilization of sample weights in latent variable models. Structural Equation Modeling A Multidisciplinary Journal, 6(4), 305321.

Kallis G, Kiparsky M, Norgaard R (2009) Collaborative governance and adaptive management: lessons from California's CALFED Water Program. Environmental Science \& Policy. 12 (6), 631-643.

Pirsoul N, Armoudian M (2019) Deliberative democracy and water management in New Zealand: a critical approach to collaborative governance and co- 
management initiatives. Water Resources Management.33,4821-4834.

Li H, Zhang XY (2016) A study on regional eco-innovation synergy and its influencing factors. China Population - Resources and Environment, 26(6), 9.

Li JQ, Xue ZM, Xie KJ (2021) Study on ecological compensation standards for interbasin water transfer projects in receiving areas. Hydropower, 47(1), 7.

Li N, Chen B (2021) The impact of fiscal policy on the synergy of civil-military integration innovation - An example of electronic and communication equipment manufacturing industry. Science and Technology Progress and Countermeasures, $38(21), 9$.

Li ZG, Liu M, Si BJ et al (2021) Research on the causes and models of sustainable supply chain co-management for water transfer projects. Science and Technology Management Research, 41(3), 9.

Lu JT (2015) Measuring and evaluating the collaborative innovation capability of Beijing-Tianjin-Hebei region - based on the composite system synergy model. Science and Technology Management Research, 35(24), 7.

Ma X (2019) Evaluation of Beijing-Tianjin-Hebei regional economic synergy based on the composite system synergy model[J]. Industrial Technology and Economics,38(05):121-126.

Peng ZY (2018) Multidimensional value assessment of water resources for water transfer projects. (Doctoral dissertation, Donghua University).

Qian J, Cai SB (2017) Exploring water pricing issues for inter-basin water transfer projects. Water Resources and Hydropower Express.

Saaty T (2004) Decision making - the analytic hierarchy andnetwork processes (ahp/anp). Systems Science and Systems Engineering, 13(1), 1-1.

Sun HK, Wei YQ (2003) The design scheme of automation system of Dahuofang 
reservoir water transmission project. Water Resources Technical Supervision.

Tang LY, Zhou JL, Wang GH (2013) Research on the evaluation of integrated innovation of industry-university-research based on dematel-anp - An example of Dalian double d port enterprises. Science and Technology Management, 34(12), 10.

Wang FW, Xu YS, Wang ZK et al (2021) DEMATEL-ANP-based selection of safety indicators for major component ships sailing in high winds and waves. Journal of Safety and Environment (01), 62-69. doi:10.13637/j.issn.1009-6094.2019.1374.

Wang HM, Zhang LL, Wang ZZ et al (2004) A review of supply chain-based feasibility study on water allocation and dispatch of the South-North Water Transfer East Route. Water Economics, 22(3), 4.

Wang LD (2006) Selection of management mode and evaluation of management performance of cross-basin water transfer project construction. (Doctoral dissertation, Hohai University).

Wang MY, Liu Y, Li MM et al (2020) Construction and application of a model for evaluating the synergy of regional carbon emission reduction capacity. Systems Engineering Theory and Practice, 40(2), 14.

Wang WQ (2013) On the synergy of regional emergency linkage. Exploration and Controversy, 1(005), 50-53.

Wang XC, Wu M (2019) Reflections on strengthening the protection and management of water sources in the South-North Water Transfer Central Line. Journal of the Yangtze River Academy of Sciences, 36(9), 5.

Wang ZF (2017) A collaborative mechanism for emergency information of the SouthNorth Water Diversion Central Project based on Spark framework. Industrial Safety and Environmental Protection (06), 27-31. 
Wang ZG, Ren XW (2019) Research on the relationship between the synergy between agricultural industrial clustering and urbanization on agricultural economic growth - A case study in Shandong Province. Agricultural Technology and Economics (3), 13.

Xie KJ (2017) Research on ecological compensation standards in the middle line receiving area of South-North Water Diversion Project. (Doctoral dissertation, North China Electric Power University; North China Electric Power University (Beijing)).

Xu LX, Cheng M (2013) A study on chinese regional scientific innovation efficiency with a perspective of synergy degree. Technology and Investment, 04(4), 229235.

Yao N (2018) A study on supply chain synergy of manufacturing enterprises based on game fuzzy element model. (Doctoral dissertation, North China Electric Power University).

Yin P (2018) Research on the evaluation of cross-border e-commerce supply chain synergy. (Doctoral dissertation, Jiangsu University of Science and Technology).

Yin W, Wang C, Xin XK (2020) Water quality management in the South-North Water Diversion Main Trunk Canal: Issues and considerations. People's Yangtze River, $51(3), 8$.

You JJ, Lin PF, Wang J et al (2018) Research on the coupling method of water allocation and scheduling for cross-basin water transfer projects. Water Resources and Hydropower Technology, 49(1), 7.

Zhang S (2016) Research on social governance performance evaluation of reservoir migrant villages in the South-North Water Diversion Central Project. Journal of China Institute of Water Resources and Hydropower Science (2), 7. 
Zhao LM, Jiang YZ, Li B (2006) Design and implementation of a comprehensive evaluation system for water resources allocation in the basin. South-North Water Transfer and Water Conservancy Science and Technology, 4(1), 3.

Kallis G, Kiparsky M, Norgaard R (2009) Collaborative governance and adaptive management: lessons from California's CALFED Water Program. Environ. Sci. Pol. 12 (6), 631-643. 